\title{
Automatic Microcalcification Detection Using Wavelet Transform
}

\author{
Ayman A. AbuBaker
}

\begin{abstract}
This paper proposes a method that can enhance the performance of Computer Aided Diagnosis (CAD) by automatically detecting and classifying the microcalcifications (MCs) in mammogram image accurately and efficiently using multi statistical filters and wavelet decomposition transform. The proposed method is divided to two main stages. In first stage, the potential MCs region (PMR) is detected based on visual characteristics of the MCs in the mammogram images. Then wavelet decomposition transform is implemented to classify the PMR to true positive and false positive regions based on extraction four wavelet features for the mammogram image. This novel method was found to be sensitive in detecting MCs in mammogram images by achieving a high true positive percentage of $98.1 \%$ and a low false positive rate 0.63 cluster/image for both MIAS and USF databases.
\end{abstract}

Index Terms-Mammogram, microcalcifications, wavelet transform, wavelet features.

\section{INTRODUCTION}

Breast cancer is the most common type of cancer among women [1]. Some important signs of breast cancer that radiologists are seeking to find are microcalcifications (MCs), masses, and structural disorders. MCs are observed in mammograms as white spots varying in size and shape. Mammography as a screening tool is one of the best proven technique for early breast cancer detection. Mammographic image analysis is a complicated and difficult task which requires opinion of highly trained radiologists. Detection of MCs, a possible symptom of breast cancer is a complex task because of the inhomogeneous background and the high noise level in the images due to emulsion artifacts. The MCs has important characteristics in their size, shape/morphology, amount, and distribution. Their sizes vary from $0.1 \mathrm{~mm}$ to 1 $\mathrm{mm}$ [2]. MC detection is very difficult in mammographies with overlapping breast tissues or high breast tissue density. Moreover, low contrast MCs can be perceived as noise while comparing them with the nonhomogeneous background. MCs are observed in mammograms individually or in clusters. Clustered MCs are more likely to be malignant. A cluster is defined as a group consisting of 3 or more MCs in a $1-\mathrm{cm}^{2}$ area. As proposed in this study, many computer-aided detection systems have been developed for MCs.

There are problems with the subjective analysis of mammographic images by radiologist. Subjective analysis depends mainly of the experience of the human operator, but

Manuscript received February 4, 2014; revised April 16, 2014.

Ayman A. AbuBaker is with the Electrical and Computer Engineering Department, Applied Science University, Amman, Jordan (e-mail: a_abubaker@asu.edu.jo). it is also affected by fatigue and other human-related factors. Since, the interpretation is a repetitive task that requires lot of attention to minute details, it requires lot of staff time and effort, which results in increasing diagnosis time. On the other hand, the objective analysis of mammograms, which is carried out by automated systems, provides consistent performance but its accuracy is usually lower. Due to the sensitivity of this problem, I believe that radiologists should be involved and computers should not replace them completely. However, computer systems can help them perform better by enhancing the quality of images, highlighting the suspicious regions and providing better analysis tools.

For these reasons, computer-aided diagnoses (CAD) are exciting a great deal of attention from the radiologist community [3], [4]. CAD is defined as a diagnosis made by a physician taking into account the computer output as a second opinion. The goal of applying CAD is to support radiologists' image interpretation and improve the diagnostic accuracy and consistency [4], [5].

Many authors have implemented a variety of CAD algorithms to detect the MCs in the mammogram images, with a range of success. This paper presents a new algorithm that can detect the MCs in the mammogram images accurately. This algorithm uses several unique characteristics of MCs to segment the potential MC regions. Then four features are generated using the wavelet transform in order to increase the sensitivity of the proposed detection algorithm

This paper is organized as follows. A brief survey of previous work is presented in Section II. A brief description for the used data based is presented in Section III. Where section IV presents the materials and methods that are used in this proposed approach. The results, discussion and evaluation are presented in Section $\mathrm{V}$, while concluding remarks are given in Section VI.

\section{RELATED WORK}

Several authors have investigated multi-scale approaches such as the wavelet transform to detect the MCs in the mammogram images. For example, Lemaur et al. [6] proposed a technique using wavelets and their Sobelev regularity index. The detection rate was slightly improved by using a modified Matzinger polynomials wavelet instead of a traditional wavelet. The algorithm performance was tested using TP and FP parentages. So they detected $66.67 \%$ TP clusters with 8.7\% FPF. Songyang and Guan [7] devised an algorithm to detect the MCs in mammogram images using wavelet features and neural networks (NNs). They proposed to employ the fourth level of a Daubechies-orthogonal 
wavelet family using features including the median contrast and normalized gray level as inputs to train a feed-forward NN classifier. Wavelet features are considered while training the NN in order to trace the likelihood map that shows the possibility of a pixel value corresponding to an MC. The resulting algorithm was successful in detecting $94 \%$ of the mean TP at the cost of one FP per image and $90 \%$ of the mean TP detection rate at the cost of 0.5 FPs per image. Rafayah et al. [8] proposed a computer aided diagnosis algorithm using a wavelet analysis and fuzzy-neural approach for detecting the MCs in mammogram images. During feature extraction, feature vectors are determined for horizontal, diagonal and vertical coefficients and normalization of the coefficients and energy and feature reductions carried out. Two classifiers were generated: one globally processed using a neuro-fuzzy classifier and the other locally processed through a cropped region of interest (RoI). Sung-Nien et al. [9] proposed a hybrid method that used a wavelet transform and a Markov random field model in order to detect the MCs in mammogram images. At first, all suspicious MCs are preserved by thresholding a filtered mammogram via a wavelet filter according to the mean pixel value (MPV) of that image. Subsequently, Markov random field parameters based on the Derin-Elliott model are extracted from the neighborhoods of all suspicious MCs as the primary texture features. The primary features combined with three auxiliary texture quantities serve as inputs to classifiers for the recognition of true MCs in order to decrease the FP rate. As a result, the detected TP was $92 \%$ with $0.75 \mathrm{FP}$ cluster per image. Sentelle et al. [10] investigated a rapid, multiresolution-based approach combined with wavelet analysis to provide an accurate segmentation of potential MCs. An initial multiresolution approach to fuzzy c-means (FCM) segmentation was employed to rapidly distinguish medically significant tissues. Tissue areas chosen for high-resolution analysis are later broken into multiple windows. Within each window, wavelet analysis is performed to generate a contrast image, and a local FCM segmentation generates an estimate of the local intensity. A simple two-rule fuzzy system then combines intensity and contrast information to derive fuzzy memberships of pixels in the high-contrast, bright pixel class. A double threshold is finally applied to this fuzzy membership to detect and segment MCs. This approach was implemented on 25 images obtained from the Digital Database for Screening Mammography (DDSM), also known as USF database. The algorithm is accurately detects $94 \%$ TP clusters with 17 FP clusters per image.

Wei et al. [11] suggested a microcalcification classification using an adaptive support vector machine (SVM). They improved the classification performance from 0.78 to 0.82 with regard to the area under the ROC curve. Jona et al. [12] proposed optimization of the feature set using hybrid of Particle Swarm Optimization (PSO) and Genetic Algorithm (GA) which called Genetical Swarm Optimization (GSO) in Digital Mammograms. Their results show that GSO convergence is better than both PSO and GA. The GSO based SVM (GSO-SVM) classifier showed superior performance with accuracy of $94 \%$ that was approximately $1 \%$ higher than GA based SVM (GASVM) and PSO based

\section{SVM (PSO-SVM) classification.}

Xinsheng Zhang and Hua Xie [13] utilized Contourlet Transform, Generalized Gaussian Mixture Model (GGMM) and Bayesian classifier to enhance the suspicious features. Hu et al. suggested a detection Algorithm [14] of Suspicious Lesions by Adaptive Thresholding Based on Multi resolution Analysis along with morphological filter to remove the noise and to enhance the gray-level feature and shape feature. Wiselin G. [15] utilized features from wavelet decomposition and Gabor filter for detecting micro calcification using back propagation neural network. Manimegalai et al. [16] developed a system extracting statistical features by wavelet decomposition for classifying breast tissue using Back propagation Neural Network (BPNN). Leena Jasmine [17] used a new approach for micro calcification detection using back propagation neural network and non-subsampled Contour let transform which yielded a significant true detection rate approximately $87 \%$.

\section{DATABASE RESOURCES}

In this work, the MC detection algorithm is trained and tested on 190 mammographic images from the University of South Florida (USF) and MIAS databases (140 from USF and the remainder from MIAS). The USF database is a publicly available digital database for mammography screening. Its images are collected from different medical schools and hospitals across the USA. These images all have the same specification $(3000$ pixel $\times 4500$ pixel and 16 - bit pixel depth). This database is divided into four volumes representing the different types of diagnosis: normal, cancer, benign, and benign without call back. Normal images are from patients with normal examination results that have had normal examinations in the previous four years. A normal screening examination is one in which no further "work-up" is required. Cancer images are from patients with screening examinations in which at least one pathology proven cancer is found. Benign cases are from patients with screening examinations in which something suspicious was found, which turned out to be non-malignant (by pathology, ultrasound or some other means). The term benign without call-back is used to identify benign cases in which no additional X-rays or biopsies were done. In this paper 70 MCs mammogram images are collected from seven cancer volumes and 70 normal mammogram images are collected from four normal volumes. The cancer volumes are: cancer_01, cancer_05, cancer_06, cancer_07, cancer_13, cancer_14, and cancer_15. The normal volumes are: normal_02, normal_05, normal_07 and normal_09.

The MIAS mammograms have been carefully selected from the United Kingdom National Breast Screening Program. The 322 images represent 161 patients in the MIAS database. These images have been expertly diagnosed and the positions of the MCs in each image are recorded.

In this paper, $25 \mathrm{MC}$ and 25 normal additional mammogram images were selected from the MIAS database. The mammograms in this database were obtained using the medio-lateral oblique (MLO) view and were digitized at a spatial resolution of $0.05 \mathrm{~mm}$ pixel size with 8-bit density resolution. Four image sizes, corresponding to different 
breast sizes, are included in the 322 images from 161 patients: small (4320 pixel $\times 1600$ pixel), medium $(4320$ pixel $\times 2048$ pixel), large (4320 pixel $\times 2600$ pixel $)$ and extra-large $(5200$ pixel $\times 4000$ pixel). Digitization was performed on a Joyce-Loeble scanning microdensitometer (SCANDIG-3) which has a linear response in the range 0.0 to 3.2 optical densities.

\section{INTELLIGENT CAD SYSTEM}

This section presents the intelligent technique that is used to detect and classify the MCs in the mammogram images. The novel technique is divided into two main sections: detection potential MCs region (PMR) and PMR classification to true and false positive regions using wavelet decomposition transform. Fig. 1 shows the flowchart for the proposed automated CAD system.

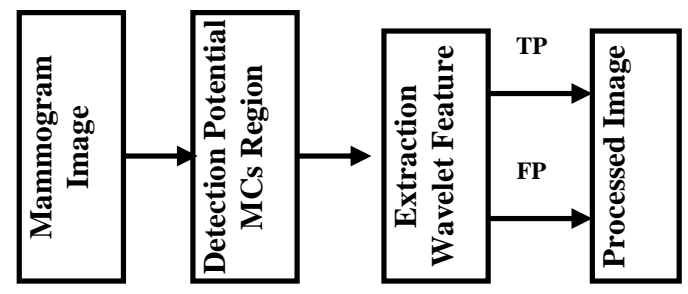

Fig. 1. Automated CAD system flowchart.

\section{A. Detection Potential MCs Region}

MCs appear on digitized mammograms as small regions, with intensity values higher than their surrounding background. The size of MCs is usually less than $1 \mathrm{~mm}$ [18]. In this paper, two concentric masks are used as shown in Fig. 2 for the initial detections of these regions. When centered on an $\mathrm{MC}$, the inner masked region included the $\mathrm{MC}$ while the outer masked region included the surrounding region.

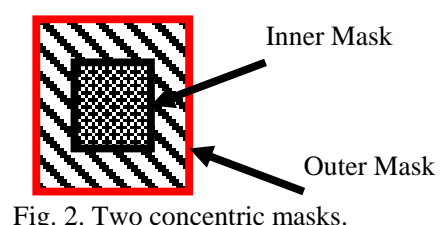

The size of the inner mask in pixels is determined by the similar resolution of the USF and MIAS databases which are $45 \mu \mathrm{m} \times 45 \mu \mathrm{m}$ and $50 \mu \mathrm{m} \times 50 \mu \mathrm{m}$ respectively. Because a rescaling process is applied in a pre-processing stage as in [19], a mask size $9 \times 9$ should be suitable to initially detect the MCs. This mask has been tested on 190 mammogram images used previously and found that all MCs are detected. A large number of non MCs regions are detected as well, which are handled in the sections to follow.

On the other hand, the average value of the outer masked region, excluding the central region, is used to investigate the surrounding region and compare it with the average of inner masked region. To find the optimum size for the outer mask, four different mask sizes were investigated as following: $13 \times$ $13,15 \times 15,17 \times 17$, and $19 \times 19$. After processing $650 \mathrm{MCs}$ clusters from 190 USF and MIAS mammogram images, it was found that the outer mask of size $13 \times 13$ is the best discrimination between the averages of the inner masks.
For a region to be selected as potential MCs region (PMR) for further MC processing, the following conditions must be satisfied:

The pixel at the centre of the inner mask should have a greater value than the average of its neighbour's pixels inside this mask as shown in Fig. 3.

Also the average value of the central region should be greater than the average value of the outer region as shown in Fig. 3.

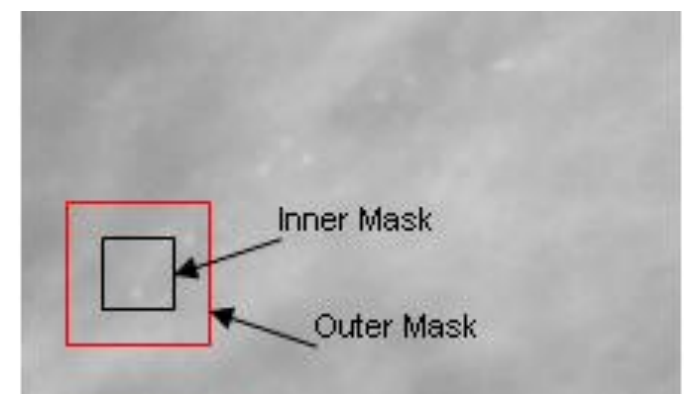

(A). The inner and outer mask surrounding an MC.

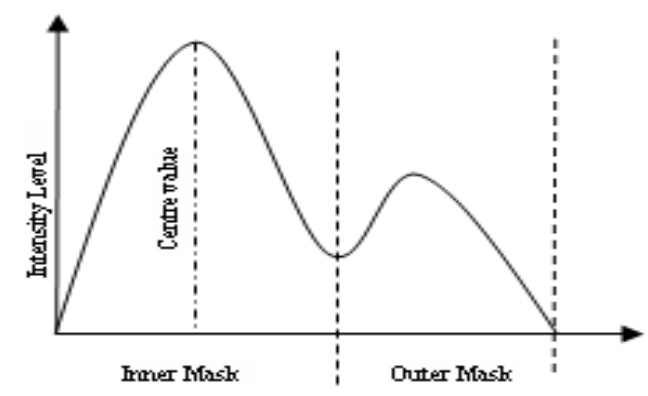

(B) the intensities of the inner and outer masks.

Fig. 3. Detection the bright regions based on the inner and outer masks.

As a result, the PMR algorithm can accurately detect all MCs in the mammogram images as shown in Fig. 4. All the MCs in the mammogram images are detected but with large number of false positive (FP) clusters. This in case reduces the sensitivity of the proposed CAD system. Therefore, the regions grouping and wavelet transform are used to reduce number of detected FP clusters and increase the sensitivity of this CAD system.
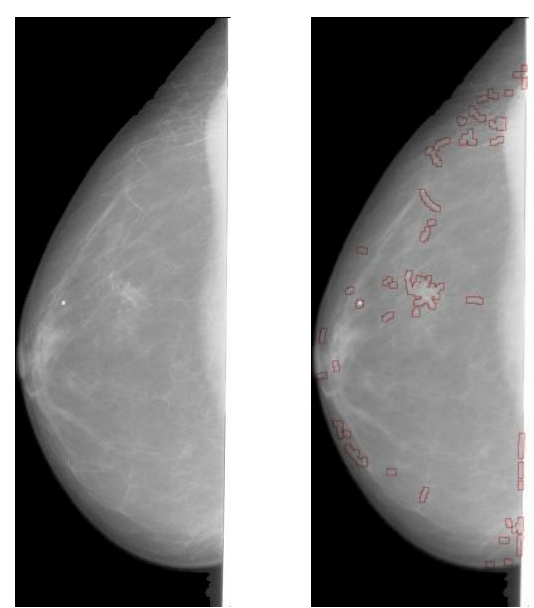

(A) the original image $\quad$ (B) the processed Image Fig. 4. PMR processing result.

\section{B. False Positive Cluster Reduction}

Reducing the detected FP clusters is processed in two phases. The fact that the MCs in the mammogram images are 
localized regions is used as a phase one. Then four wavelet features are generated to classify the detected regions as true and false positive clusters is used a second phase. The following subsections present these two phases that are used in reducing the detected FP clusters.

\section{1) Grouping of microcalcifications}

Usually, the MCs diagnosed by an expert are clustered in localized regions of the mammogram image [20]. There should be at least 2 to three clusters in a small local region according as in [21]. Therefore, the distances between the detected regions by the proposed PMR algorithm are considered as the final criterion for accepting these regions as MCs or not, as shown in Fig. 5. These distances are set to be $2 \mathrm{~mm}$ (20 pixels) in the horizontal and vertical directions between the detected centers. Hence, if an MC is found between these two centers, it will be highlighted as potential MCs.

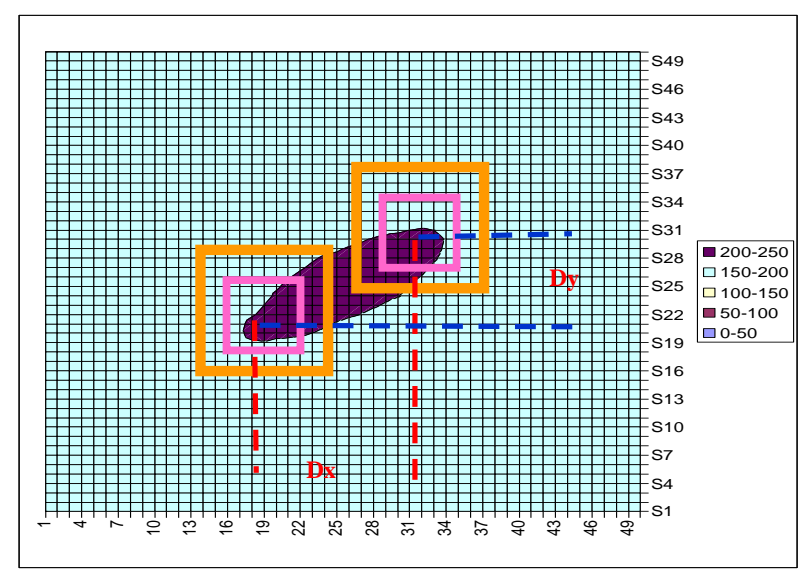

Fig. 5. Measurement of the distance between two detected MCs. The two end points of the elongated vascular MC shown will be detected and associated with an MC cluster.

\section{2) Extraction wavelet features}

The wavelet decomposition is introduced as a next stage to classify the detected PMR to false positive and true positive regions. Due to the nature of MCs clusters, which appear as a bright region within a mammogram, they also appear as peak discontinuities for the wavelet transform [22]. This makes the wavelet transform appropriate to classify MCs and for feature generation as it is argued that wavelets have finite square supports and are best in capturing point discontinuities and not edges [23].

The Wavelet coefficients are divided into low (L) frequency approximation coefficients and high $(\mathrm{H})$ frequency detail-coefficients. The high frequency coefficients are further divided into detail sub-bands: vertical (LH), horizontal (HL), and diagonal (HH) coefficients. The low frequency (LL) approximation-coefficients provide a reduced resolution representation of the original image which can be transformed again according to the wavelet level applied. Applying wavelet decomposition to an image will produce an approximation matrix that is a quarter of the original area of an image.

Fig. 6 shows the default view of wavelet decomposition with the features extracted from the top left corner, which is the low frequency image from the second level of decomposition. This provides a better view of the differences between levels of decomposition especially when looking at the low frequency image that produces the approximation coefficients for generated features. The figure shows the original image at the top and below it are the approximations from level 1 and level 2 of decomposition showing mass lesion in a larger area than from the original image.

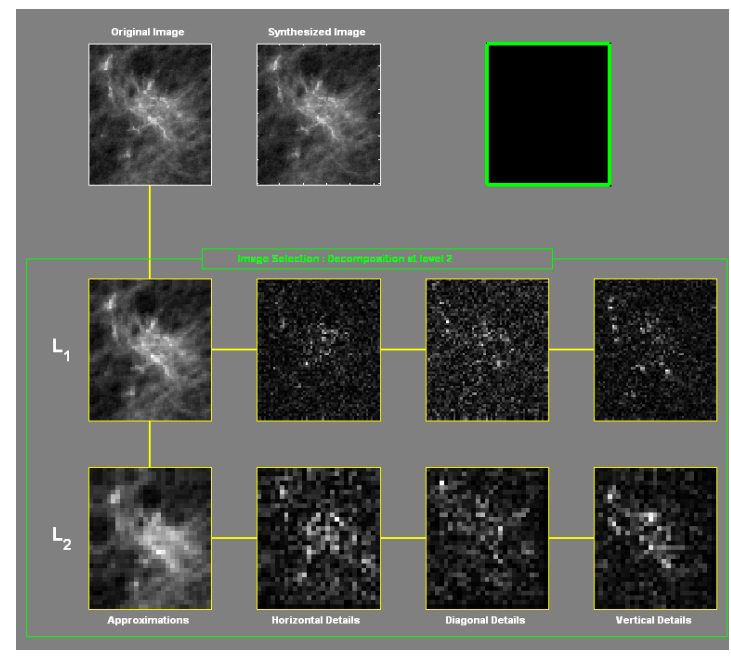

Fig. 6. DB4 decomposition wavelet at level 2.

In this paper, the wavelet Daucechies (BD4) transform is used to generate the wavelet coefficient. These coefficients will be used to classify the detected regions to TP and FP regions.

The wavelet DB4 is implemented on the segmented region of interest images of size $13 \times 13$ produced by the previous stage. Then, four main features are generated based on the maximum 100 wavelet coefficients. These coefficients are collected from the coefficients of the low frequency image. These features are maximum value of the coefficients, minimum value of the coefficients, average value of the coefficients, and standard deviation between the coefficients. These features are extracted from low frequency image at Daucechies (BD4) with level 4 as shown in Fig. 7.

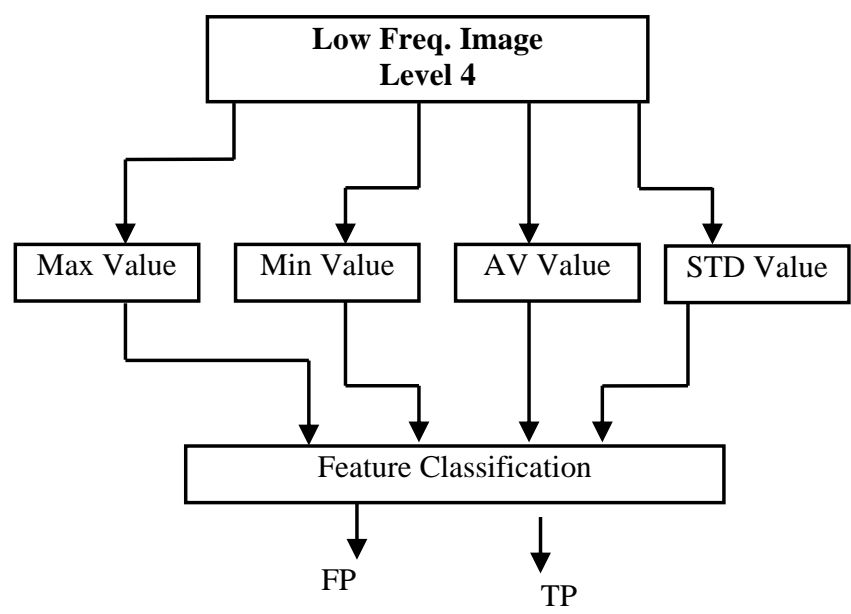

Fig. 7. Extraction wavelet features flowchart.

\section{Algorithm Evaluation}

The automated MCs detection algorithm was applied first using 190 MC mammogram images from the USF and MIAS 
database. The processed images were later subjectively compared with pre-diagnosis cases for the mammogram images from the databases in order to classify the detected regions into TP and FP clusters as shown in Fig. 8. Using these classified results, the TP and FP comparisons with other authors was carried out and is shown in Table I. It is worth mentioning that the TP and FP rates in these authors' publications are reported for different mammogram images and use different benchmarks.

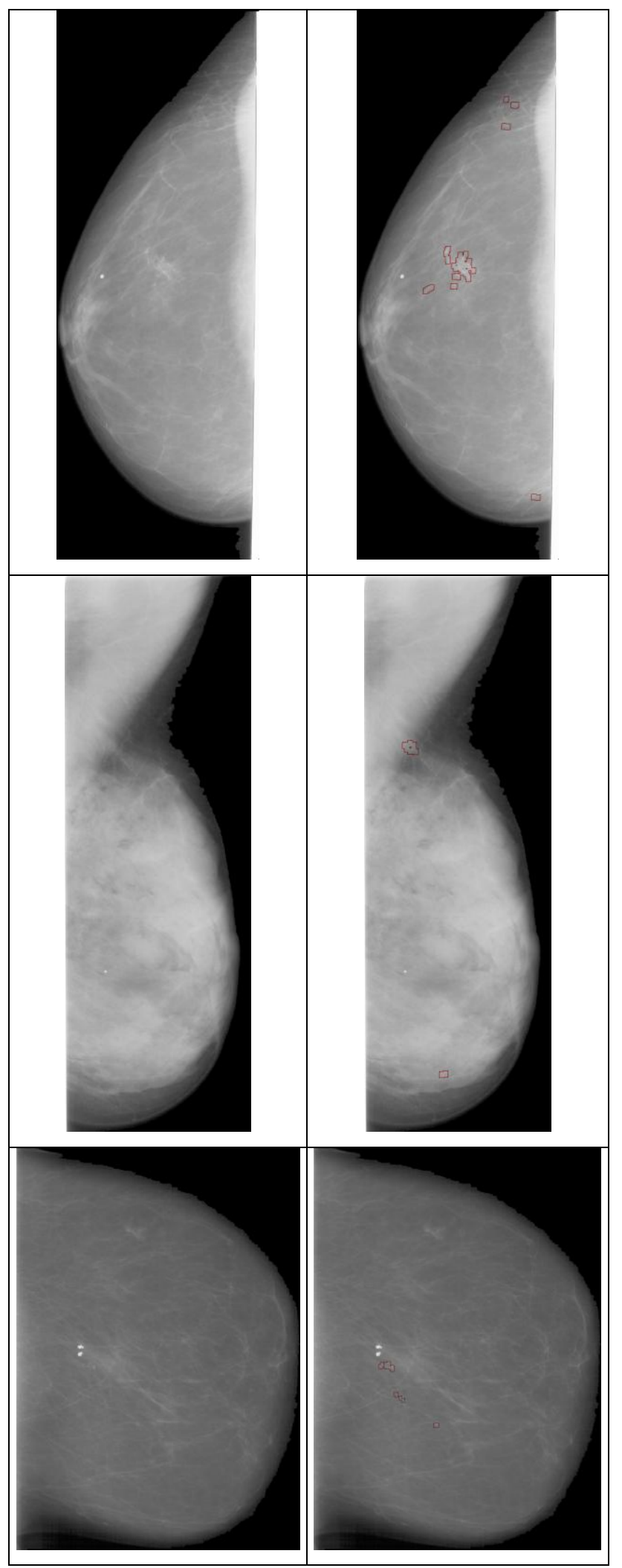

Fig. 8. Accurate detection of MC clusters.
TABLE I: THE COMPARISON RESULTS USING OTHER AUTHOR'S ALGORITHMS

\begin{tabular}{c|clc}
\hline No. & Algorithm Name & $T P$ & FP \\
\hline 1. & Sentelle [10] & 94 & 17 per image \\
2. & Zhang, discriminate [12] & 70 & NA \\
3. & Zhang, logistic regression [12] & 70 & NA \\
4. & E. Malar[20] & 94 & NA \\
5. & Mohanalin, [21] & 96.55 & 0.4 per Image \\
6. & Brijesh [24] & 85 & NA \\
7. & Linguraru [25] & 91 & 0.95per image \\
8. & Peng, KD-GA [26] & 98.9 & $40 \%$ FPF \\
9. & Peng, GA [26] & 85 & 20\%FPF \\
10. & Rizzi [27] & 98 & 1 per Image \\
11. & Ravi [28] & 91 & 1.63 per Image \\
12. & Oliver [29] & 80 & 1 per Image \\
\hline 13. & The Proposed Algorithm & 98.1 & 0.63 per image \\
\hline
\end{tabular}

From Table I, it can be seen that the proposed MC detection algorithm achieves good overall performance compared to other techniques in detecting and classifying the TP and FP regions. The TP percentage achieved for the automatic detection of MC lesions in mammograms from both MIAS and USF databases is about $98.1 \%$ with a FP rate of about 0.63 clusters/ mammogram.

\section{CONCLUSION}

The new novel method was designed to diagnose and detect MCs using several MC characteristics. This CAD system includes two main stages: detecting potential MC region in the mammogram image, then reducing the detected FP clusters using region grouping and wavelet features. In the first stage, multi statistical filters are applied to detect the potential MCs regions in the mammogram image which results many false positive regions. The next stage is designed based on MCs visual characteristics in which at least 2 to three MC clusters should be in a small local region. Therefore, the region grouping is firstly applied to reduce the detected FP clusters. Then, four wavelet features are generated in order to reduce the FP clusters. This algorithm is tested on 190 mammogram images from both USF and MIAS database. As a result, this algorithm can detect the MCs accurately in mammogram images with average detection rates of about $98.1 \%$ for true-positives and about $0.63 \mathrm{FP}$ clusters/image, when tested on both USF and MIAS databases.

\section{ACKNOWLEDGMENT}

The author is grateful to the Applied Science Private University, Amman, Jordan, for the financial support granted to cover the publication fee of this research article.

\section{REFERENCES}

[1] S. P. Sajda, C. Spence, and J. Pearson, "Learning contextual relationships in mammograms using a hierarchical pyramid neural network," IEEE Transactions on Medical Imaging, vol. 21, no. 3, pp. 135-141, 2002.

[2] L. Bocchi, G. Coppini , J. Nori, and G. Valli, "Detection of single and clustered microcalcifications in mammograms using fractals models and neural networks," Journal of Medical Engineering \& Physics, vol. 5, no. 3, pp. 230-238, 2002.

[3] K. Doi, H. MacMahon, S. Katsuragawa, R. M. Nishikawa, and Y. Jiang, "Computer-aided diagnosis in radiology: Potential and pitfall," Eur. J. Radiol., vol. 31, pp. 97-109, 1999. 
[4] A. F. Laine, J. Fan, and W. Yang, "Wavelets for contrast enhancement of digital mammography," IEEE Eng. Med. Biol. Mag., vol. 14, no. 5, pp. 536-550, Sept.-Oct. 1995.

[5] A. AbuBaker, R. S. Qahwaji, Musbah J. Aqel, and Mohmmad H. Saleh, "Mammogram image size reduction using 16-8 bit conversion technique," International Journal of Biomedical Sciences, vol. 1, no. 2 , pp. 103-110, 2006.

[6] G. Lemaur, K. Drouiche, and J. DeConinck, "Highly regular wavelets for the detection of clustered microcalcifications in mammograms," IEEE Transactions on Medical Imaging, vol. 22, no. 3, 2003.

[7] S.-Y. Yu and L. Guan, "A CAD system for the automatic detection of clustered microcalcification in digitized mammogram films," IEEE Transactions on Medical Imaging, vol. 19, no. 2, 2000.

[8] R. Mousa, Q. Muniba, and A. Moussa, "Breast cancer diagnosis based on wavelet analysis and fuzzy-neural," Elsevier Journal of Expert System with Applications, vol. 28, pp. 713-723, 2005.

[9] S.-N. Yu, K.-Y. B. Li, and Y.-K. Huang, "Detection of microcalcifications in digital mammograms using wavelet filter and Markov random field model," Computerized Medical Imaging and Graphics, vol. 30, pp. 163-173, 2006.

[10] S. Sentelle C. Sentelle, and M. A. Sutton, "Multiresolution-based segmentation of calcifications for the early detection of breast cancer,' Real-Time Imaging, vol. 8, pp. 237-252, 2002.

[11] L. Wei, Y. Yang, and R. M. Nishikawa, "Microcalcification classification assisted by content based image retrieval for breast cancer diagnosis," Pattern Recognition, vol. 42, no. 6, pp. 1126-1132, 2009.

[12] P. Zhang, B. Verma, and K. Kumar, "Neural vs. statistical classifier in conjunction with genetic algorithm based feature selection," Pattern Recognition Letters, vol. 26, pp. 909-919, 2005.

[13] X.-S. Zhang and H. Xie, "Mammograms enhancement and denoising using generalized gaussian mixture mode 1 in nonsubsampled contourlet transform domain," Journal of Multimedia, vol. 4, no. 6 , 2009.

[14] K. Hu, X.-P. Gao, and F. Li, "Detection of suspicious lesions by adaptive thresholding based on multiresolution analysis in mammograms," IEEE Transactions on Instrumentation and Measurement, vol. 60, no. 2, February 2011.

[15] J. Dheeba and G. W. Jiji, "Detection of Micro calcification Clusters in Mammograms using Neural Network," International Journal of Advanced Science and Technology, vol. 19, pp. 13-22, June 2010.

[16] P. Manimegalai, P. Revathy, and K. Dhanushkodi, "Microcalcification detection in mammogram image using wavelet transform and neural network," International Journal of Advanced Scientific Research and Technology, vol. 1, no. 2, February 2012.

[17] J. S. Leena. A. G. Jasmine, and S. Baskaran, "Classification of microcalcification in mammograms using nonsubsampled contourlet transform and neural network," European Journal of Scientific Research, vol. 46, no. 4, pp. 531-539, 2010.

[18] H. S. Sheshadri and A. Kandaswamy, "Computer-Aided diagnosis of digital mammograms," Journal of Information Technology, 2005.

[19] A. AbuBaker, R. S. Qahwaji, M. J. Aqel, and M. H. Saleh, "Efficient pre-processing of usf and mias mammogram images," Journal of Computer Science, vol. 4, no. 3, 2006.
[20] E. Malar, A. Kandaswamy, D. Chakravarthy, and A. G. Dharan, "A novel approach for detection and classification of mammographic microcalcifications using wavelet analysis and extreme learning machine," Journal of Computers in Biology and Medicine, vol. 42, issue 9, pp. 898-905, 2012.

[21] B. Mohanalin, P. K. Kalra, and N. Kumar, "A novel automatic microcalcification detection technique using Tsallis entropy \& a type II fuzzy index," Journal of Computers \& Mathematics with Applications, vol. 60, issue 8, pp. 2426-2432, October 2010.

[22] K. Bovis, S. Singh, J. Fieldsend, and C. Pinder, "Identification of masses in digital mammograms with MLP and RBF nets," in Proc. the IEEE-INNS-ENNS International Joint Conference on Neural Networks Com, 2000, pp. 342-347.

[23] M. P. Sampat, M. K. Markey, and A. C. Bovik, "Computer-aided detection and diagnosis in mammography," Handbook of Image and Video Processing, 2nd ed. New York: Academic Press. pp. 1195-1217, 2005.

[24] B. Verma and P. Zhang, "A novel neural-genetic algorithm to find the most significant combination of features in digital mammograms," Applied Soft Computing, vol. 7, pp. 612-625, 2007.

[25] M. G. Linguraru, K. Marias, R. English, and M. Brady, "A biologically inspired algorithm for microcalcification cluster detection," Medical Image Analysis, vol. 10, pp. 850-862, 2006.

[26] Y.-H. Peng, B. Yao, and J.-M. Jiang, "Knowledge-discovery incorporated evolutionary search for microcalcification detection in breast cancer diagnosis," Artificial Intelligence in Medicine, vol. 37, pp. 43-53, 2006.

[27] M. Rizzi, M. D'Aloia, and B. Castagnolo, "Computer aided detection of microcalcifications in digital mammograms adopting a wavelet decomposition," Journal of Integrated Computer-Aided Engineering, vol. 16, no. 2, pp. 91-103, 2009.

[28] K. R. Samala, H.-P. Chan, Y. Lu et al., "Detection of microcalcifications in breast tomosynthesis reconstructed with multiscale bilateral filtering regularization," in Proc. The International Conference of Medical Imaging 2013: Computer-Aided Diagnosis Carol L. Novak; Stephen Aylward Lake Buena Vista (Orlando Area), Florida, USA, February 09, 2013.

[29] A. Oliver, A. Torrent, X. Lladó et al., "Automatic microcalcification and cluster detection for digital and digitised mammograms," Journal of Knowledge-Based Systems, vol. 28, pp. 68-75, April 2012.

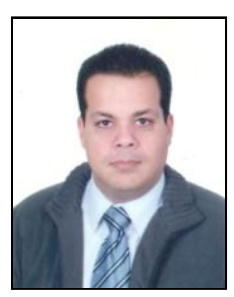

Ayman A. AbuBaker got his B.Sc. in electrical engineering in 1999. In 2008 he finished his $\mathrm{PhD}$ in electronic imaging and media communications (EIMC) from School of Informatics, University of Bradford, UK. His main research focused on processing medical images such as mammogram images (detecting microcalcifications and mass lesions), ultrasound images and MRI images. He is currently an associate professor at Electrical and Computer Engineering Dept., Applied Science Private University, Amman-Jordan. 